\title{
Current Status, Issues, and Challenges of Blockchain Applications in Education
}

\author{
https://doi.org/10.3991/ijet.v15i12.13797 \\ Yan Ma, Yiming Fang (四) \\ Shaoxing University, Shaoxing, P. R. China \\ ymfang@usx.edu.cn
}

\begin{abstract}
Due to the unique characteristics of decentralization and security, blockchain is believed to have considerable potential to provide a wide range of benefits for education development. Its application in education is relatively new but increasing very quickly. This paper introduced the typical blockchain techniques and characteristics briefly. Then, recent applications of blockchain in education were summarized comprehensively, especially those regarding learning record keeper, certificate issue and management, and decentralized education ecosystem. Finally, technical and non-technical challenges were discussed. It is hoped to provide an in-depth look at the perspectives of blockchain in evolving education and help to the development of new application systems.
\end{abstract}

Keywords-Blockchain, educational application, current status, issues and challenges.

\section{Introduction}

The blockchain, an emerging technology, was first introduced in the original paper "Bitcoin: A Peer-to-Peer Electronic Cash System" published by Satoshi Nakamoto [1]. Since then, blockchain has been widely used as a value transferring mechanism and helped prompt the successful launch of cryptocurrencies, including Bitcoin, Litecoin, Ethereum, et al. [2,3]. Now, increasingly scientists believe that this technology is in a position to become the fifth disruptive computing paradigm after mainframes, PCs, the Internet, and mobile/social networking [4, 5]. Currently, more and more applications of blockchain sprang up in various fields, and the financial industry was the typical one to find ways to use the blockchain technology. More than 300 companies joined the alliances of R3 or Ethereum for continually evolving the blockchain in banking [6]. Linq, a blockchain platform issued by the US Nasdaq, is employed to complete and record private securities transactions. It is now believed to have possibilities to evolve the traditional financial system and reduce the cost of the transaction significantly [7].

The applications of blockchain also contributed to scientific research and technological innovations in the energy field. A piloted platform on developing microgrids, LO3 Energy, was reported by Siemens aiming for local energy trading [8]. The microgrid hosted by Transactive Grid in Brooklyn, New York, has facilitated point-to- 
point transactions of energy. Residents can sell the surplus electricity generated by their solar panels to their neighbors, and the transaction was based on the blockchain network. Therefore, the transaction can be managed and recorded without the participation of personnel [9]. In the medical field, blockchain helped address the issue of the information island. With blockchain, the medical data, usually stored in different institutions, can be shared with high access security, scalability, and data privacy. It promoted effective medical treatment in turn [10]. The applications of blockchain in the food industry were reported to be promising to reduce food waste, which is an increasingly severe problem over the world [11, 12].

Blockchain has the characteristics of decentralization, verifiability, and immutability, which can offer a wide range of benefits to education development. The high potential has been demonstrated by many application cases [13, 14]. However, the blockchain research in education is rather scarce, and the application has just begun. Therefore, it is meaningful to analyze the previous studies, understand their advantages and disadvantages, and reveal their disruptive potential (i.e., which changes they might induce).

The remainder of this paper was organized as follows. Section 2 provided a short introduction of blockchain technology and its characteristics that are essential to education technology. Subsequently, typical applications of blockchain in education at present were summarized in Section 3. Section 4 discussed the issues and challenges existing, followed by Section 5, which concluded this paper.

\section{Blockchain Technology and its Characterize}

\subsection{Concept of blockchain}

In the simplest terms, blockchain can be described as a linked list of blocks. Each block within the blockchain mainly contains one block header and one block body. The block body is employed to store the transaction information. The block header is utilized to save the hash value of the previous block and then links to it. Hence, the blocks are connected into a chain on which the entire history of each transaction is recorded [15].

\subsection{Crucial technologies of blockchain}

Blockchain is a comprehensive technical system rather than a single one. Its key technologies mainly comprise consensus mechanism, encryption algorithm, smart contract, and distributed data storage $[4,16,17]$.

Consensus mechanism: Blockchain is actually a type of distributed ledger. Each participant has the right to maintain and update the database, and each one has a copy of the ledger. A consensus mechanism is a set of rules or protocols that ensure all participants are on the same page of the ledger, thus ensuring that the blockchain information stored in each node is not falsified. At present, the popular consensus 
mechanisms include proof of stake (PoS), proof of work (PoW), delegated proof of stake (DPoS), etc. [18].

Encryption algorithm: Encryption plays a vital role in encrypting the data on the blockchain. Once the encryption method is cracked, the data security of blockchain will be challenged, and the immunity of blockchain will no longer exist. The encryption algorithm usually used can be divided into symmetric encryption algorithm and asymmetric encryption algorithm. In the blockchain, the asymmetric encryption algorithm is mainly used. The encryption algorithms use "public key" and "private key" to perform encryption and decryption. When one key (public key or private key) is adopted to encrypt data, only its corresponding key, generated using a known algorithm, can be used to decrypt the data. The public keys are publicly known and essential for identification, whereas private keys are kept secret and are used for authentication and encryption. RSA, ECC and SM2 are the typical asymmetric encryption algorithms commonly used in blockchain currently [19].

Smart contract: A smart-contract is an event-driven computer program that is adopted to automate transactions. With the smart contract, the payment can be triggered automatically, and the workflow of the transactions becomes convenient, frictionless and less error-prone for the individuals. The application of smart contract can not only reduce the management cost, but also avoid unnecessary disputes, because under the smart contract. All transactions can be executed accurately [20].

Distributed data storage: All full nodes on the blockchain network are equal in hierarchy and each node acts as an autonomous agent. They store and maintain their own subsets of the shared ledger on the local space. This type of decentralized storage can address many problems found in a centralized system. It can improve the reliability, availability and access efficiency of the system, and so it is widely used in the field of blockchain [21].

\subsection{Key features of blockchain}

Briefly, the following features of blockchain are very interesting and make the technology appealing to education [3, 22, 23].

Decentralization: There is no centralized node or governing authority in the blockchain. The rights and obligations of any node are equal. The entire chain is jointly maintained by all the nodes. Transactions can be conducted between any two parties in the blockchain network, without the authentication by the central administrator.

Openness: Credible mathematical algorithms were utilized to regulate transactions on the blockchain. The rules and operation procedures are open and transparent. Most of the data stored on the blockchain, except the private information being encrypted, are completely permissionless and transparent. In addition, multiple copies of the data are stored at many nodes throughout the entire network. Anyone can access the data through the hash value of the block header. It is difficult to hide the data, so the whole system is highly transparent.

Traceability and auditability: Each transaction on the ledger is recorded with a timestamp and validated through a verified hash reference. Therefore, the transactions 
can be traced and tracked easily to their previous transactions. The audit trail that exists on the blockchain makes it easy to trace transactions back to their origins.

Security: Blockchain provides high security of information through the following three methods. Firstly, the storage with high redundancy is used to ensure the consensus of the data. Only when most of the nodes agree, can the update of the nodes be allowed. Secondly, the encryption algorithm is used to verify the data to ensure that it is difficult to modify them. Finally, the multi-private keys are utilized to control the access rights. The privacy of users can be protected while ensuring transparency and traceability of the blockchain.

\section{Recent Applications of Blockchain in Education}

Today, many types of blockchain applications in education have been investigated. In this paper, they were categorized into learning record keeper in Section 3.1, certificates issue and management in Section 3.2, and decentralized education ecosystem in Section 3.3.

\subsection{Learning record keeper}

Conventionally, education organizations are the unique keepers and authority of student learning records. This makes it quite burdensome for an employer or other interested third recipient to access and verify the "official" data. What's more, the "official" data usually exclusively covers the abbreviated course title, the course number, the letter grades, and GPA (grade point average). The potential employers can hardly know what the student has done while taking the course. The help from the official data is limited to the decision-making process [24]. Blockchain enables innovative opportunities in storing the learning records of students. Using the blockchain's built-in mechanism of smart contract, the actual work the students have done in a lab or a service-learning project, as well as the As and Bs along with the course titles, can be saved in the students' records. Employers can have a more comprehensive view of the job-hunter and thus make a better decision.

Devine proposed a novel concept of blockchain-based learning. The learning outcomes were treated as virtual coins that could be packed into blocks and placed into blockchain by teachers. Then, the entire learning record was available for all to access, including potential employers [25]. Farah presented a framework to trace the performance of students for their multi-learning activities. Learners were allowed to select one or more repositories to save their data. The distributed records can be gathered and integrated from multiple sources to support personal development and reflective learning across platforms. Thus, this learning block included all metadata about many activities [26]. Ocheja recommended a blockchain-based approach for connecting learning data between institutions and organizations. Then it was achieved that the learning data was stored and controlled with consistency, usability, immutableness, security, privacy [27]. As an educational scheme, EduRSS combined cryptography algorithms, distributed storage, and blockchain and achieved secure storage and cross- 
institutional sharing of educational records [28]. In [29], Daraghmi introduced UniChain, which was designed to manage Electronic Academic Records (EARs). The typical technologies of blockchain, such as timed-based smart contracts, encryption techniques, etc., were employed. The preliminary experimental results indicated that UniChain achieved a high efficiency for handling a large dataset at low latency. In almost all application cases, blockchain technology was considered to be ideal as a promising tool to secure, share, and verify learning records [17].

With the learning record stored, the blockchain can be expanded to evaluate the students' professional ability, which provides employment and employee selection with great convenience. In the work reported by Zhao [30], the design scheme of a student ability evaluation system was present. K-means clustering algorithm was applied to the students' academic performance and achievements that were stored on the blockchain. Objective evaluation of the student's professional ability was achieved, which would help the student employment. Wu studied a blockchain-based skill evaluation model and applied it to measure the students' professional knowledge and expertise. The feasibility was tested by simulating the teacher and student scenarios in an operational skill competition. The evaluation process was simplified significantly, and high efficiency was achieved [31]. Duan developed an automatic assessment software as a learning tool based on blockchain. All learning outcomes, including process and evidence, the indicator of the graduation requirement, the course name, and the course weight, are all saved to the block. Consequently, the evaluation can be performed according to the graduation requirement index of universities [32].

\subsection{Certificates issue and management}

Certificates are usually utilized to reveal that the learner has followed all the rules and had good behavior at school. During the several years attending school, the learners are genrally awarded various certificates, such as academic certificate, report card, student certificate, award certificate, English level certificate, professional qualification certificate, etc. It has been widely proved that the blockchain's features of security, transparency, and immutability could bring benefits to the work of issuing and managing certificates [23]. The technology enables students to directly access all kinds of certificate information they have obtained anytime and anywhere. What's more, the blockchain technology allows education institutes to manage the students' academic certificates securely and efficiently. Finally, the certificate information recorded on the blockchain will also be permanently stored in the cloud server to effectively solve the problem of authentication and enhance the credibility. Potential employers can directly validate the information provided by students.

During the past years, an increasing number of applications of blockchain were proposed to address this problem. Using the blockchain-based system of Blockcerts, the MIT Media Lab started issuing digital certificates for learners in a community in 2015. The recipients have more control over their own certificates. Then, there is no need for a verification process by any third party [33]. Another leader in blockchain education, the University of Nicosia, issued and verified digital certificates in a completely decentralized way. All graduates received a PDF copy of the diploma on the 
blockchain in addition to the normal physical one [34]. Similarly, a permissioned blockchain-based platform, named 'CredenceLedger', was proposed for decentralized verification of academic credentials. Techniques of blockchain ledger were employed to store the compact data proofs of digital academic credentials. All the credentials were protected employing blockchain's inherent security characteristics and can be easily verified for education stakeholders and interested third-party intermediaries [35]. EduCTX, a credit platform derived from the open-source Ark Blockchain Platform, also provided a distributed and interoperable architecture model to process, manage, and control the credits (named as ECTX tokens) that students gained for completing courses. HEIs (high education institutions) had opportunities to access to more up-to-date data of the students regardless of their educational origins, and students are allowed for a transparent and single view of their completed courses [36].

\subsection{Decentralized education ecosystem}

Blockchain is a decentralized network that does not have any central authorities. All stakeholders have the full copy of the ledger, and each participant has the right to validate updates to the chain. The feature provides a collaborative environment for all parties, including learners, teachers, and authorities. All the parties can benefit from the technique [37].

In the view of education institutes, the decentralization feature helps to improve the management efficiency of schools. Sony Global Education announced to provide global educational services beyond the traditional framework. It reduced the intervention of education administrative departments in the evaluation of the learning process and results, made education fairer, and greatly improved the efficiency of education management [38]. Bore introduced a novel applicant case of blockchain for School Information Hub (SIH). A blockchain-based system was utilized to collect, validate and manage the data about schools in Kenya. As a result, it would be easy for the decision-makers to know the student level and what was happening at a school. Then better decisions can be made and more appropriate interventions can be designed [39].

The teaching can also benefit a lot from the application of blockchain. For example, effective communication among teachers and learners is vital for E-learning. A novel interactive multimedia system was provided to encourage teachers and students to establish effective communication. Differently, the data exchange was supported by blockchain and its decentralized topology. The authors believed that the interaction among teachers and learners could run with high efficiency, security, and privacy with this implemented system [40]. Zhong proposed a novel application of proposed in Elearning. According to the incentive policies deployed on the blockchain network, the system they developed can give an extra reward to the top-ranked learners in the form of virtual currency. With this solution, the learning engagement was claimed to be improved [41]. In the aforementioned system proposed by Duan, the evaluation of students' achievement in university can be converted to the post-job competency evaluation, and the student competency evaluation can also be projected to the teaching evaluation of each curriculum, which helped the curriculum's continuous improvement [32]. 


\section{$4 \quad$ Issues and Challenges of Blockchain in Education}

Even though the blockchain technology has a high possibility for disrupting the education system, it is still in its experimental beginnings and has to face a number of challenges. Some of them come from the immature technology and some others come from the non-technological factors.

\subsection{Technological issues}

Firstly, scalability is one of the main problems published in many literatures [15, 23]. A more significant number of the blockchain nodes causes a more massive storage demand and lower propagation speed in the network. The mechanism of synchronous storage requires all participants to store transaction records and related information. The users have to provide mass storage systems for it. Meanwhile, with the increasing number of the blockchain nodes, the efficiency of the consensus algorithm, as well as the response speed of the nodes, reduce significantly.

Secondly, security is another obstacle that blockchain education has to overcome. Even though the blockchain is inherently capable of providing a secure solution, threats from within and without still exist [42]. In theory, only when more than $51 \%$ of nodes are attacked and controlled by hackers at the same time can data information be leaked or tampered with. However, with the development of mathematics, cryptography and computing technology, it is difficult to guarantee that the algorithm will not be cracked in the future, resulting in the leakage of teachers' and students' information [43]. Moreover, all transactions are open and transparent, and any information can be traced and inquired. Then some conclusions can be inferred, or the state and behavior of teachers and students can be predicted, which is not conducive to the protection of teachers' and students' privacy.

\subsection{Non-technological factors}

1. There is a severe shortage of skilled blockchain engineers and experts. In recent years, with the promotion and application of blockchain in many industries, the demand for human resources of blockchain has been increasing. Both the companies in the growing period and those well-known ones that desire to catch up with this technology are looking for engineers for developing a blockchain-based system. However, to develop a secure and reliable blockchain education system with full function and high usability, a high-level R \& D group is needed. Notably, the members are expected to have a solid technical and mathematical foundation, which poses a big problem for the practitioners and scholars in the education field. Blockchain technologies, platforms, and smart contracts are not easy to understand by learners, educators, and other professional parties in the chain [29, 44]. It seems to be a solution to assign the development task to computer engineers. Unfortunately, the system designed by computer engineers often cannot satisfy the needs of education participants very well. 
2. At present, most researches on blockchain focus on disrupting the traditional financial industry and the application in education are extremely new. Further development faces the challenges of lack of policy protection and practical experience. First, due to the lack of policy protection and guidance, the decentralization will have a substantial impact on traditional education institutions. The conventional education platform suffered heavy losses, causing relevant institutions and departments to take a negative attitude to the new applications. Secondly, due to the small number of application cases, experts and scholars are wait-and-see and lack the motivation to promote further uses.

3. Due to the decentralization, the data on the blockchain is distributed recorded and stored on the blockchain, which makes the property rights of students' data blurred. Currently, the school affairs office is generally responsible for data management. With the application of blockchain, all data were stored on the blockchain, making the responsibilities of the entity management department be downplayed. Then new problems appear. Who owns the virtual data? Who owns the right to use? Who owns the results based on data analysis? The series of the issues caused by the data property rights have to be studied and addressed for the further application of blockchain in education.

\section{Conclusion}

The development and application of blockchain technology have created new technical foundations and practical models for education and teaching reform. Although many issues and challenges still exist, such as the construction, maintenance and management of blockchain technology infrastructure and platform, the application, ownership and privacy protection of students' learning data, etc., there is no doubt that, from traditional primary and secondary schools to universities and educational administrative institutions, and then to business enterprises engaged in education and training industry, all have realized the huge potential of blockchain technology. Therefore, they have invested resources such as funds, technology, and talents for investigating the education innovation using blockchain.

Presently, the application of blockchain in education is still in the nascent stage. The application scope is also very limited -- mainly focusing on learning record keeping, certificates issue and management, and decentralized education ecosystem building. It is expected that educational institutions, teachers, educational researchers and learners will hold an open and positive attitude to the impact and changes and made corresponding preparations for the extensive and in-depth application of blockchain technology.

\section{Acknowledgement}

This study was supported by the China Postdoctoral Science Foundation (2019M662112) and the Scientific Research Foundation of Shaoxing University 
(20195024). The authors gratefully acknowledge the anonymous reviewers for their valuable comments.

\section{$7 \quad$ References}

[1] Satoshi Nakamoto S. (2016). Bitcoin: A peer-to-peer electronic cash system, https://bitcoin.org/en/bitcoin-paper https://doi.org/10.1007/978-3-030-17740-9_3

[2] Casino, F., Dasaklis, T. K., Patsakis C. (2019). A systematic literature review of blockchain-based applications: Current status, classification and open issues. Telematics and Informatics, 36: 55-81. https://doi.org/10.1016/j.tele.2018.11.006.

[3] Yumna, H., Khan, M. M., Ikram, M., Ilyas, S. (2019). Use of blockchain in education: A systematic literature review. Asian Conference on Intelligent Information and Database Systems (ACIIDS 2019), April 8-11 2019, Yogyakarta, Indonesia. pp 191-202. https://doi. org/10.1007/978-3-030-14802-7_17

[4] Wu, J., Tran, N. K. (2018). Application of blockchain technology in sustainable energy systems: An overview. Sustainability, 10 (9): 3067. https://doi.org/10.3390/su10093067.

[5] Swan, M. (2015). Blockchain: Blueprint for a new economy. O'Reilly Medi.

[6] Clincy, V., Shahriar H. (2019). Blockchain development platform comparison. IEEE 43rd Annual Computer Software and Applications Conference (COMPSAC), July 15-19 2019, Milwaukee, WI, USA. pp 922-923. https://doi.org/10.1109/COMPSAC.2019.00142.

[7] Parker, L. (2015). Nasdaq Linq claims to have issued first securities over a blockchain, https://bravenewcoin.com/insights/nasdaq-linq-claims-to-have-issued-first-securities-overa-blockchain.

[8] Deign, J. (2016). Siemens joins the growing blockchain bandwagon for peer-to-peer energy sales, https://www.greentechmedia.com/articles/read/siemens-joins-growing-blockchain -bandwagon.

[9] (2016). Blockchain transactive grid set to disrupt energy trading market, https://www. engerati.com/energy-retail/blockchain-transactive-grid-set-to-disrupt-energy-tradingmarket/. https://doi.org/10.1109/pesgm.2018.8586099

[10] Chen, Y., Ding, S., Xu, Z., Zheng, H., Yang, S. (2018). Blockchain-based medical records secure storage and medical service framework. Journal of Medical Systems, 43, Article number: 5. https://doi.org/10.1007/s10916-018-1121-4.

[11] Kamilaris, A., Fonts A., Prenafeta-Boldú, F. X. (2019). The rise of blockchain technology in agriculture and food supply chains. Trends in Food Science \& Technology, 91: 640-652. https://doi.org/10.1016/j.tifs.2019.07.034.

[12] Borah, M. D., Naik, V. B., Patgiri, R., Bhargav, A., Phukan, B., Basani S. G. (2019). Supply chain management in agriculture using blockchain and IoT, Advanced Applications of Blockchain Technology, Springer. pp 227-242. https://doi.org/10.1007/978-981-13-8775-3 11.

[13] Sosa, E., Salinas, J., De Benito B. (2017). Emerging technologies (ETs) in education: A systematic review of the literature published between 2006 and 2016. International Journal of Emerging Technologies in Learning (iJET), 12(5): 128-149. https://doi.org/10.39 91/ij et.v12i05.6939.

[14] Sun, H., Wang, X., Wang, X. (2018). Application of blockchain technology in online education. International Journal of Emerging Technologies in Learning (iJET), 13(10): 252259. https://doi.org/10.3991/ijet.v13i10.9455. 
[15] Zheng, Z., Xie, S., Dai, H. N., Chen, X., Wang, H. (2018). Blockchain challenges and opportunities: A survey. International Journal of Web and Grid Services, 14 (4): 352-375. https://doi.org/10.1504/IJWGS.2018.10016848.

[16] Yli-Huumo, J., Ko, D., Choi, S., Park, S., Smolander, K. (2016). Where is current research on blockchain technology?-a systematic review. PLoS ONE, 11(10), e0163477. https://doi. org/10.1371/journal.pone.0163477.

[17] Grech, A., Camilleri, A. F. (2017). Blockchain in education. JRC science for policy report, Luxembourg, Publications Office of the European Union. https://doi.org/10.2760/60649.

[18] Du, M., Ma, X., Zhe, Z., Wang, X., Chen, Q. (2017). A review on consensus algorithm of blockchain. 2017 IEEE International Conference on Systems, Man, and Cybernetics (SMC), October 5-8 2017, Banff, Canada. pp 2567-2572. https://doi.org/10.1109/SM C.20 17.8123011.

[19] Halaburda, H. (2018). Blockchain revolution without the blockchain?. Communications of the ACM, 61 (7): 27-29. https://doi.org/10.1145/3225619.

[20] Sayeed, S., Marco-Gisbert, H., Caira, T. (2020). Smart contract: Attacks and protections. IEEE Access, 8: 24416-24427. https://doi.org/10.1109/ACCESS.2020.2970495.

[21] Raman, R. K., Varshney, L. R. (2018). Distributed storage meets secret sharing on the blockchain. Information Theory and Applications Workshop (ITA), October 25 2018, San Diego, CA. pp 1-6. https://doi.org/10.1109/ITA.2018.8503089.

[22] Kamišalić, A., Turkanović, M., Mrdović, S., Heričko, M. (2019). A preliminary review of blockchain-based solutions in higher education. Learning Technology for Education Challenges (LTEC 2019), July 15-18 2019, Zamora, Spain. pp 114-124. https://doi.org/10. 1007/978-3-030-20798-4_11.

[23] Alammary, A., Alhazmi, S., Almasri, M., Gillani, S. (2019). Blockchain-based applications in education: A systematic review. Applied Sciences, 9(12): 2400. http://doi.org/10. 3390/app9122400.

[24] Han, M., Li, Z., He, J., Wu, D., Xie, Y., Baba, A. (2018). A novel blockchain-based education records verification solution. 19th Annual SIG Conference on Information Technology Education (SIGITE '18), October 3-6 2018, Fort Lauderdale. pp 178-183. https://doi. org/10.1145/3241815.3241870.

[25] Devine, P. (2015). Blockchain learning: can crypto-currency methods be appropriated to enhance online learning?. ALT Online Winter Conference 2015, December 7-10 2015. pp 1-33. http://oro.open.ac.uk/44966/1/Devine2015-altc-blockchainlearning.pdf.

[26] Farah, J. C., Vozniuk, A., Rodríguez-Triana, M. J.., Gillet, D. (2018). A blueprint for a blockchain-based architecture to power a distributed network of tamper-evident learning trace repositories. IEEE 18th International Conference on Advanced Learning Technologies (ICALT), July 9-13 2018, Mumbai India. pp 1-5. https://doi.org/10.1109/ICALT.20 18.00059 .

[27] Ocheja, P., Flanagan, B., Ogata, H. (2018). Connecting decentralized learning records: a blockchain based learning analytics platform. 8th International Conference on Learning Analytics and Knowledge (LAK '18), March 5-9 2018, Sydney Australia. pp 265-269. https://doi.org/10.1145/3170358.3170365.

[28] Li, H., Han, D., Li, K. C., Castiglione, A. (2019). EduRSS: A blockchain-based educational records secure storage and sharing scheme. IEEE Access, 7: 179273-179289. https://doi. org/10.1109/ACCESS.2019.2956157.

[29] Daraghmi, E. Y., Daraghmi, Y. A., Yuan, S. M. (2019). UniChain: A design of blockchain-based system for electronic academic records access and permissions management. Applied Sciences, 9(22): 4966. https://doi.org/10.3390/app9224966. 
[30] Zhao, W., Liu, K., Ma, K. (2019). Design of Student Capability Evaluation System Merging Blockchain Technology. Journal of Physics: Conference Series, 1168: 032123. https:// doi.org/10.1088/1742-6596/1168/3/032123.

[31] Wu, B., Li, Y. (2018). Design of evaluation system for digital education operational skill competition based on blockchain. IEEE 15th International Conference on e-Business Engineering (ICEBE), October 12-14 2018, Xi'an, China. pp 102-109. https://doi.org/10.110 9/ICE BE.2018.00025.

[32] Duan, B., Zhong, Y., Liu, D. (2017). Education application of blockchain technology: Learning outcome and meta-diploma. IEEE 23rd International Conference on Parallel and Distributed Systems (ICPADS), December 15-17 2017, Shenzhen, China. pp 814-817. https://doi.org/10.1109/ICPADS.2017.00114.

[33] Jones, B. (2017). MIT has started issuing diplomas using blockchain technology, https://futurism.com/mit-has-started-issuing-diplomas-using-blockchain-technology.

[34] (2018). Issue and verify blockchain-based certificates, https://www.decentralized.com/blo ck-col.

[35] Arenas, R., Fernandez, P. (2018). CredenceLedger: A permissioned blockchain for verifiable academic credentials. IEEE International Conference on Engineering, Technology and Innovation (ICE/ITMC), June 17-20 2018, Stuttgart, Germany. pp 1-6. https://doi.org/10. 1109/ICE.2018.8436324.

[36] Turkanović, M., Hölbl, M., Košič, K., Heričko, M., Kamišalić, A. (2018). EduCTX: A blockchain-based higher education credit platform. IEEE Access, 6: 5112-5127. https:// doi.org/10.1109/ACCESS.2018.2789929.

[37] Wanotayapitak, S., Saraubon, K., Nilsook, P. (2019). Process design of cooperative education management system by cloud-based block-chain e-portfolio. International Journal of Online and Biomedical Engineering (iJOE), 15(8): 4-17. https://doi.org/10.3991/ijoe.v15i0 8.10374.

[38] (2016). Sony Global Education Develops Technology Using Blockchain for Open Sharing of Academic Proficiency and Progress Records, https://www.sony.net/SonyInfo/News/ Press/201602/16-0222E/.

[39] Bore, N., Karumba, S., Mutahi, J., Darnell, S. S., Wayua, C., Weldemariam, K. (2017). Towards blockchain-enabled school information hub. The 9th International Conference on Information and Communication Technologies and Development (ICTD '17), November 16 - 19 2017, Lahore, Pakistan. pp 1 - 4. https://doi.org/10.1145/3136560.3136584.

[40] Bdiwi, R., Runz, C. d., Faiz, S., Cherif, A. A. (2017). Towards a new ubiquitous learning environment based on blockchain technology. IEEE 17th International Conference on Advanced Learning Technologies (ICALT), July 3-7 2017, Timisoara, Romania. pp 101-102. https://doi.org/10.1109/ICALT.2017.37.

[41] Zhong, J., Xie, H., Zou, D., Chui, D. K. (2018). A blockchain model for word-learning systems. 5th International Conference on Behavioral, Economic, and Socio-Cultural Computing (BESC), November 12-14 2018, Kaohsiung, Taiwan. pp 130 - 131. https://doi.org/ 10.1109/BESC.2018.8697299.

[42] (2019). Top five blockchain security issues in 2019, https://ledgerops.com/blog/2019/03/ 28/top-five-blockchain-security-issues-in-2019.

[43] Lin, I. C., Liao, T. C. (2017). A Survey of Blockchain Security Issues and Challenges. International Journal of Network Security, 19(5): 653-659. https://doi.org/10.6633/ IJNS.201 709.19(5).01.

[44] Ocheja, P., Flanagan, B., Ueda, H., Ogata, H. (2019). Managing lifelong learning records through blockchain. Research and Practice in Technology Enhanced Learning, 14, Article number: 4. https://doi.org/10.1186/s41039-019-0097-0. 


\section{Authors}

Yan Ma received a master's degree in 2013 from Zhejiang A \& F University, Hangzhou, China. She majors in the Agriculture Informationization, and her current research focuses on the emerging technologies on education management. She has been working at Business School, Shaoxing University, Shaoxing, China.

Yiming Fang received a Master degree in Computer Application Technology from Hangzhou Dianzi University, Hangzhou, China, in 2006, and a Ph. D. degree in Agricultural Mechanization Engineering from Nanjing Agriculture University, Nanjing, China, in 2017. He is currently an Associate Professor with the School of Mechanical \& Electrical Engineering, Shaoxing University, Shaoxing, China. His research interests include applications of emerging computer technologies. (E-mail: ymfang@usx.edu.cn)

Article submitted 2020-02-16. Resubmitted 2020-03-28. Final acceptance 2020-03-30. Final version published as submitted by the authors. 